\title{
LAS BIOGRAFÍAS MÉDICAS: ¿ESTUDIO DE CASOS PARA UNA INTRODUCCIÓN (HISTÓRICA) A LA MEDICINA?
}

\author{
Carmen Ruiz García \\ Profesora Titular de Historia de la Ciencia, Universidad de Córdoba
}

\begin{abstract}
RESUMEN
El trabajo discute el papel pedagógico de las biografías en la Introducción a la Medicina y Metodología Científica. Concluimos que es un complemento didáctico interesante en el conjunto del proceso educativo, si bien su uso exclusivo es insuficiente para proporcionar una visión de conjunto sobre los conocimientos y las prácticas médicas a lo largo de la historia en su triple dimensión: científica, social y cultural.
\end{abstract}

PALABRAS CLAVE: Biografías médicas, educación medica e historia, recursos didácticos en Historia de la Medicina.

\section{SUMMARY}

This paper discuss the didactic role of biographical method for the subject Introduction to Medicine and Scientific Methodology. We suggest to use medical biographies as a complementary tool of global training process, although an exclusively use of this is inadequate to provide an integrated view, as well scientific as social or cultural approaching, about the medical knowledge and practice in the past.

KEY WORDS: Medical biographies, history in medical education, teaching strategies in History of Medicine.

¿Qué aporta la historia a la enseñanza de la Medicina? Se trata de una pregunta que ha tenido y tiene respuestas variadas. Entre otras, una disciplina «puente entre las Humanidades y la Ciencia», materia que «da una visión de conjunto de la Medicina», asignatura que «contribuye a la aclaración de problemas actuales de la Medicina mostrando la génesis de los mismos en tiempos pasados y contextualizándolos en el entorno social, político y cultural», «contribuir a la comprensión sociológica de saberes, prácticas y problemas 
médicos de forma que permita clarificar y solucionar cuestiones planteadas en la Medicina», etc., etc. ${ }^{1}$ Una visión que recoge elementos de prácticamente todas las ideas anteriores y que resulta más próxima a nosotros en el tiempo es la que nos proporciona la Declaración Institucional de la Sociedad Española de Historia de la Medicina que, entre otras cosas, afirma que la Historia de la Medicina es la «única disciplina que proporciona una mirada unitaria sobre los conocimientos y las prácticas que la conforman, en sus dimensiones científica, social y cultural» ${ }^{2}$. En definitiva, diríase que la Historia de la Medicina trata de ofrecer al estudiante una visión de conjunto que compense el enfoque fraccionado del ser humano y de la Medicina que ofrece el resto de las materias que componen la licenciatura, proporcionando de este modo un panorama histórico general de los principales momentos del desarrollo de la Medicina.

Rosen enseña que un curso de Historia de la Medicina, desde el punto de vista pedagógico, debe mostrar, por una parte, el desarrollo de la medicina como totalidad y, por otra, enfrentarse con el problema del cambio a través del análisis de los elementos que lo configuran: políticos, económicos, sociales, religiosos, culturales ${ }^{3}$. Las diversas orientaciones relativas al contenido que debe tener un programa de Historia de la Medicina han estado relacionadas muy directamente con los grandes paradigmas de la investigación histórico-médica. De forma esquemática puede hablarse de tres grandes modelos tradicionales de investigación histórico-médica con técnicas peculiares perfectamente definidas: el Bio-bibliográfico, el Filológico, y el Institucional. A lo largo del siglo XX se han desarrollado otros dos modelos: el Históricocultural y el Histórico-social ${ }^{4}$.

La biografía médica es un género historiográfico muy antiguo, anterior incluso a la propia disciplina y que tiene una larga tradición historiográfica.

1 Una visión más detallada la encontramos bajo el título «El lugar de la historia en la educación médica», en Rosen, G. (1985), De la policía médica a la medicina social, México, Siglo XXI, pp. 12-49.

2 La Historia de la Medicina ante el proceso de integración del sistema universitario español en el espacio europeo de enseñanza superior. XII Simposio de la SEHM. Alicante, 2627 de junio de 2003. http://balmis.umh.es/shm/declaracion.PDF

3 Rosen, G. (1968), «What Medical History should be taught to medical students», en Blake, J.B. (ed.) Education in the History of Medicine, New York-London, Hafner Publishing, pp. 19-27.

4 Estos dos modelos a diferencia de los tradicionales, carecen de recursos técnicos unívocos, ya que el historicocultural depende básicamente de reformulaciones de los métodos filológicos, y el historicosocial de los propios del modelo institucional asociados a los procedentes de las diversas ciencias sociales. 
Este género es el primer acercamiento a la Historia de la Medicina que se inició, como es sabido, en el mundo islámico con la aparición de repertorios bio-bibliográficos redactados por tratadistas 5 . Pero en cuanto que recurso pedagógico, el género biográfico puede constituir un intento de exaltación patológica de lo individual en la enseñanza de la Historia de la Medicina. Supone un riesgo que hay que evitar, ya que podríamos hacer creer a los alumnos que el progreso científico se debe exclusivamente a la existencia de grandes figuras a las que habría que venerar, reverenciar, admirar, etc., en una trágica caricatura de lo que la Historia de la Medicina es y debe ser; juicio que mereció a Juan Luis Carrillo el problema con ocasión de la Reunión celebrada en 1988 sobre «la enseñanza de la Historia de la Medicina» ${ }^{6}$.

Con independencia de los diccionarios y repertorios bio-bibliográficos, se han escrito obras que abordan una historia biográfica de la Medicina. Por ejemplo la realizada por Sigerist ${ }^{7}$, en la que se recogían medio centenar de figuras médicas de todos los tiempos; desde el semilegendario Imhotep hasta el contemporáneo William Osler. Constituye una selección de biografías de personajes «cuyos trabajos han sido decisivos para la ciencia, trabajos que marcan una dirección y crean una escuela, imprimen su sello a una época» ${ }^{8}$. Otra biografía colectiva de la Historia de la Medicina es la publicada en 2002 por Simmons ${ }^{9}$; que abarca desde la Medicina antigua hasta los investigadores actuales del VIH como Robert Gallo y Luc Montagnier, incluyendo algunas mujeres. Una obra en la que se narra la historia de la Medicina occidental con las vidas de sus figuras más influyentes, siendo éstas elegidas en función de su importancia para la medicina contemporánea. Nuland ${ }^{10}$ publicó en 1988

5 El primero de ellos desde el punto de vista cronológico, es el Kitab al-Fibrist realizado por el tratadista árabe Ibn An-Nadín (c. 936-995). Vid.: RIERA, J. (1976), «La historiografía médica. Desde la antigüedad hasta nuestro siglo», en LAín EnTRALgO, P. (dir.), Historia Universal de la Medicina, Barcelona, Salvat. Vol. VII, pp. 448-450.

6 CARrillo Martos, J. L. (1988), «Pedagogía por objetivos. Estrategias didácticas y métodos de evaluación». En: BALLESTER AÑON, R. (coord), La enseñanza de la Historia de la Medicina. Problemas y perspectiva, Alicante, Instituto de Ciencias de la Educación, Universidad de Alicante, pp. 4-18 (p. 6)

7 Sigerist, H. E. (1949), Los grandes médicos. Historia Biográfica de la Medicina, Barcelona, Ediciones AVE.

$8 \quad$ Ibidem p. 8.

9 Simmons, J. G. (2002), Doctors and discoveries: Lives that created today's medicine, Boston, Houghton Mifflin.

10 Nuland, S.B. (1988), Doctors: The Biography of Medicine, New York, Alfred Knopf. Existe una traducción al francés: NulAND, S. B. (1989), Les héroes de la medecine. Paris, Cop. 
una obra, que lleva por subtítulo La biografía de la medicina, en la que se aborda una historia de la profesión a través de 14 de sus figuras más destacadas. En nuestro ámbito Laín ${ }^{11}$, en un intento de «dar a la historia de la medicina la anchura y la profundidad que la medicina y la historia por sí mismas poseen» publicó, bajo el título Grandes Médicos una obra en la que se recogen las biografías de Paracelso, Vesalio, Harvey, Bichat, Laennec, Ramón y Cajal, y von Weizsäcker. Por último, entre otros podríamos citar Los diez mayores descubrimientos de la medicina ${ }^{12}$. Como señala su prefacio, proporciona un relato íntimo de la vida de los científicos que fueron los responsables, según el autor, de los diez descubrimientos más significativos desde 1543: «sin estos fundamentos indispensables, la medicina, tal y como hoy la conocemos y la practicamos, no sería posible». La finalidad de estas obras no parece ser la programación docente de una disciplina como la de Historia de la Medicina. En algún caso, los autores tratan de completar esos contenidos, o bien su intención es casi exclusivamente divulgativa, aunque estén realizadas a veces con rigor.

En lo que a la enseñanza de la Historia de la Medicina en nuestro país y dentro de nuestra área de conocimiento, la única docencia que actualmente se imparte con las características del modelo biográfico se lleva a cabo en la Universidad de la Laguna. Se trata de una asignatura de libre elección bajo la denominación de «Grandes Médicos» con dos créditos teóricos. Pero como es sabido, se anuncian grandes cambios en el marco universitario europeo, que básicamente consisten en una reformulación conceptual del currículo de la educación superior mediante su adaptación a los nuevos modelos de formación centrados en el trabajo del estudiante. El protagonista del proceso educativo pasará a ser el estudiante, quien de manera crítica deberá aprender a establecer relaciones y a fijar nociones, transformándose en un ente activo de modo que el centro del proceso cognitivo radique en su propio esfuerzo. Qué duda cabe de que dicho modelo afectará a nuestros actuales patrones de enseñanza de la Historia de la Medicina.

En este nuevo contexto universitario, las biografías de los científicos en general y de los médicos en particular puede ser útil para enseñar Historia de la Medicina. No como un cuerpo exclusivo de conocimientos, sino como una aproximación al conocimiento de la medicina, un conocimiento en construcción capaz de poner de manifiesto la pluralidad de posiciones respecto al pa-

11 Lain Entralgo, P. (1961), Grandes Médicos, Barcelona, Salvat.

12 Friedman, M.; Friedland, G.W. (1999), Los diez mayores descubrimientos de la medicina, Barcelona, Paidós. 
sado, y su mayor o menor fundamentación metodológica. Es aplicable, en este sentido, lo que señala J. L. Barona en relación a la historiografía: no debemos perder de vista que el enfoque biográfico no posee un valor intrínseco en sí, pero es cierto que puede aportar ejemplificaciones importantes para el estudio global de los problemas de la Historia de la Medicina considerados en un ambiente histórico concreto ${ }^{13}$. No debe olvidarse, por otra parte, que en el discurso biográfico está en juego la tensión entre la singularidad del individuo concreto, con la trayectoria vital única, irrepetible y, como no, sujeta al azar, y la representatividad como exponente de una determinada época o, quizás, de un movimiento o proceso en la historia de la medicina ${ }^{14}$. Obviamente, por ello es necesario tener una visión de conjunto. Si nos limitamos tan sólo a biografías, difícilmente podremos ofrecer al alumnado una panorámica general y básica de la Historia de la Medicina, puesto que la Historia de la Medicina, no puede reducirse a la reunión o síntesis de una serie de biografías científicas; o si lo prefieren, no puede reducirse a una cadena lineal de personajes con ideas brillantes. El mito no explica, sólo justifica. Y el presente no requiere justificación, puesto que ya existe; lo que necesita es una explicación ${ }^{15}$.

Hay que tener en cuenta otra serie de factores que no pueden ser reducidos a la escala individual. Es importante que hagamos ver al alumnado que «los avances específicos atribuidos a una persona no se producen por la acción de una sola persona, sino que son hijos del clima de opinión y de los conocimientos de la época, tanto en general como del problema concreto» ${ }^{16}$. Emplear la biografía constituye un riesgo, ya que el relato hagiográfico subraya el elemento individualista en la Historia de la Ciencia, es decir, ver a la mente individual como agente único del desarrollo científico al luchar con los problemas científicos. No obstante, la biografía también añade un elemento humanizador que a menudo se echa en falta en las historias de teorías o cien-

13 Barona, J.L. (1994), Ciencia e Historia. Debates y tendencias en la historiografia de la ciencia, Valencia, Seminario de Estudios sobre la Ciencia, p. 218.

14 Así respondían MARTíNEZ y PARDO a la pregunta: ¿el progrés científic segueix unes lleis ineludibles $\mathrm{i}$ els canvis que experimenten els sabers $\mathrm{i}$ les pràctiques mèdiques obeeixen a uns factors socials deterninants més enllà del protagonisme individual, aleshores quin valor presenta en aquest context la narració de la vida d'un individu concret? MARTíNEZ VIDAL, A.; PARDo TOMÁs, J. (2004), «Les Biografies Mèdiques. Entre l'hagiografia y la història», XIII Congreso de Historia de la Medicina Catalana. Tendencies historiografiques de la Medicina a les portes del segle XXI. En: http://einstein.uab.es/suab237/w/cat/news.htm.

15 HeRnÁndeZ García, G. (2002), «Historia de la ciencia y enseñanza de la ciencia». Correo del Maestro, 72. En: http://correodelmaestro.com/anteriores/2002mayo/incert72.htm.

16 RHODES, Ph. (1985), Introducción a la Historia de la Medicina, Zaragoza, Acribia, p. VII. 
cias particulares. Aún así, como queda dicho, el aspecto individualista puede llevar a exagerar el genio singular dejando de lado una explicación histórica más completa.

El uso de biografías científicas es hoy una de las tendencias más innovadoras en educación de distintas disciplinas durante la segunda enseñanza ${ }^{17}$. En este caso, el método biográfico se justifica porque los expertos en cada materia y contexto entienden que las biografías de personajes característicos pueden ser útiles para conocer un determinado proceso, ciertos avances técnicos, etc., destacar la capacidad de invención y sentar las bases para el aprendizaje técnico deseado. En la enseñanza superior, la trayectoria personal de los «protagonistas» de la historia, de sus motivaciones y de las circunstancias en que llevaron a cabo sus aportaciones, es considerada parte integrante de la Historia de la Ciencia, que es una materia troncal u optativa de las licenciaturas de ciencias, tecnología y profesiones sanitarias. Incluye, como parte sustancial, la discusión y clarificación de las ideas que motivaron la creación de los conceptos, de las circunstancias que dieron lugar a su creación o su evolución, de la justificación de su aceptación o rechazo, así como el conocimiento de las consecuencias de todo ello, tanto en la Medicina y en las Ciencias, como en la vida social, económica y política de las sociedades.

Considerando todo lo anterior, ¿cómo responder al interrogante que figura en el título de mi exposición: las biografías médicas: ¿estudio de casos para una introducción (histórica) a la medicina?

Las biografías médicas no parecen ser adecuadas como procedimiento exclusivo para desarrollar la materia troncal Introducción a la Medicina y Metodología Cientifica (cuyos descriptores son Historia de la salud, de la enfermedad y del ejercicio médico; Teoría y Método de la Medicina; Información, Documentación y Terminología Médicas; Bioestadística; Física Médica). El estudio de casos es, pues, una metodología más, que puede cumplir una función en el conjunto del proceso educativo, pero no es suficiente para comprender en todas sus dimensiones los hechos sociales y elaborar explicaciones generales.

17 Entre otras cabe citar: Solbes, J. ;TRAVER, M. (2001), «Resultados obtenidos introduciendo Historia de la Ciencia en las clases de física y química: mejora de la imagen de la Ciencia y desarrollo de actitudes positivas», Enseñanza de las Ciencias, 19 (1), 151-162. GuZMÁN, M. (1993), Tendencias innovadoras en educación matemática. En: http://www.oei.es/edumat.htm. MARTÍNEZ NAVARRO, F. Utilización didáctica en la enseñanza de la fisica y química de bachillerato de la biografia y producción cientifica de investigadores eminentes. En: http://webpages.ull.es /users/apice/pdf/124-028.pdf. REPETTO JIMÉNEZ, E.; MARTínEZ NAVARRO. Utilización de las biografias de los científicos en la enseñanza de las ciencias con una orientación de ciencia, tecnología y sociedad. En: http://webpages.ull.es/users/apice/pdf/211-029.pdf. 
Pero existen otras fórmulas pedagógicas en las que el uso de las biografías sí puede resultar de interés para el estudiante, como en las actividades académicamente dirigidas o Seminarios, que tengan por objeto, precisamente, elaborar biografías de personajes relevantes en el campo de la medicina, relacionando su obra con el contexto histórico y las aportaciones científicas.

¿Qué características debería tener la enseñanza-aprendizaje basada en las biografías médicas?

Respecto a los objetivos, este recurso didáctico debe cumplir los cinco siguientes: 1) Complementar los contenidos teóricos tratados en clase, pasando de los conocimientos generales al análisis de elementos más particulares; 2) Hacer patente la forma peculiar de aparecer las ideas en Medicina; 3) Enmarcar temporal y espacialmente las grandes ideas y/o problemas, junto con su motivación, precedentes, etc.; 4) Señalar los problemas abiertos de cada época y su evolución, 5) Apuntar las conexiones históricas de la Medicina con otras Ciencias.

Respecto a la selección de biografías, creemos que debe de ofrecer una visión tan amplia como sea posible, de distintos aspectos históricos relacionados con la salud, la enfermedad y la formación y el ejercicio de aquéllas personas que a lo largo de la historia se han ocupado de estos temas con distinto grado de profesionalización. Incluyendo, no sólo médicos, sino también otros científicos o figuras sanadoras que en el pasado ayudaron a construir la Medicina. En este sentido se podrían abordar las biografías médicas en varios bloques, con las consideraciones o exigencias siguientes. 1) Representativos de cada época. Es imposible tratar biografías que abarquen toda la constitución y desarrollo de la medicina científica. Por tanto, es necesario realizar una selección de los más representativos de cada una de las etapas históricas de la medicina. 2) Representativos de la medicina española. Teniendo en cuenta que la mayoría de las grandes figuras pertenecen a otros países, sería interesante abordar la biografía de algún/os personaje/s que sean representativos de la medicina española en distintas épocas. 3) Figuras recientes del siglo XX. 4) Desde una perspectiva de Género. La ciencia tradicionalmente ha sido una institución marcadamente androcéntrica y las mujeres hemos sido silenciadas por la historia tradicional. Las causas, entre otras, se corresponden con unas concepciones estrechas de la Historia general y de la Historia de la Ciencia en particular, que reconstruyen el periodo sobre los nombres de grandes personajes y teorías o prácticas exitosas y dejan de lado otras actividades y contribuciones en modo alguno colaterales al desarrollo de la ciencia en general o la medicina en particular. A través de este recurso pedagógico podemos dar una visión de la práctica médica realizada por mujeres, y sacar de la sombra a 
algunas de éstas que, a pesar de las dificultades, desempeñaron un papel en la ciencia y la tecnología a lo largo de las diferentes épocas históricas. Hacerlas visibles al alumnado resaltando tanto su formación, contribución científica y trayectoria profesional como sus opiniones, intereses y perfil humano ${ }^{18}$.

En cuanto a la metodología, señalamos los siguientes aspectos en el procedimiento general. A. Recopilación de la información. Debemos aprovechar el interés del alumnado hacia el uso del ordenador y el disfrute que les ocasiona la navegación por internet. Un instrumento de trabajo que puede facilitar el aprendizaje de la historia de la profesión que han elegido. B. Elaboración de una ficha de trabajo. Tras la recopilación de la información se propone elaborar una ficha de trabajo que incluya: 1) el perfil biográfico, destacando los principales aspectos de su vida y de su obra. 2) formación científica. 3) la medicina y la sociedad de su época. 4) aportaciones a la medicina. 5) implicaciones de sus aportaciones. 6) bibliografía. C. Exposición pública de resultados. Los estudiantes expondrán de forma oral al conjunto de los alumnos el resultado de su trabajo. Con dicha actividad conseguiríamos, entre otros, tres objetivos básicos que corresponden a tres competencias transversales fundamentales: aprender a trabajar en equipo, buscar y seleccionar información, $y$, aprender a exponerla oralmente.

18 Entre otros muchos trabajos al respecto, cabe destacar la propuesta didáctica realizada en SÁNCHEZ GONZÁLEZ, MD. (1999), «Las Biografías de las científicas en la enseñanza de las ciencias: mostrando una tradición», en Barral, M.J.: Magallón, C.; Miqueo, C.; Sánchez, M.D.: (eds.) Interacciones ciencia y género. Discursos y prácticas científicas de mujeres, Barcelona, Icaria, pp. 259-290. En esta propuesta se aborda la vida de Dorothy M. Crowfoot Hodgking. 